\title{
Towards Zero Packet Loss with LISP Mobile Node
}

\author{
Musab Isah $^{\infty}$, Steven Simpson ${ }^{\ell}$, Yusuf Sani ${ }^{\partial}$, Chris Edwards ${ }^{\sharp}$ \\ School of Computing and Communications (Infolab21), \\ Lancaster University, UK. \\ (m.isah ${ }^{\infty}$, s.simpson ${ }^{\ell}$, y.sani ${ }^{\partial}$, c.edwards $\left.^{\sharp}\right) @$ lancaster.ac.uk
}

\begin{abstract}
Host mobility protocols such as LocatorIdentifier Separation Protocol Mobile Node (LISP-MN) are known to experience packet loss at the point of handover. For the duration of the handover, packets sent to the MN via the old access link are dropped by the router since it has no way of knowing where the device has moved to. This affects the performance of transport layer protocols of the TCP/IP stack, which results in degradation of network performance. Buffering these packets close to the MN's new location and forwarding them to the MN on handover completion is one way of improving the overall performance of the mobility protocol. Hence, we introduce a novel network node, locserver, to buffer these packets in order to mitigate the packet loss and reduce the service disruption time (SDT) experienced by MNs during handovers. Using a laboratory testbed implementation, LISP-MN with locserver support shows significant reduction in packet loss and reduced SDT in comparison to vanilla LISP-MN. Similarly, performance analysis of DASH video player also shows the new architecture helps in improving the average video quality downloaded by the $\mathrm{MN}$ and reduces the player's instability.
\end{abstract}

Keywords-LISP; LISP-MN; locator/identifier split, mobility

\section{INTRODUCTION}

Mobile devices, especially smartphones, are increasingly becoming the primary source of access to the Internet as the number of wireless connected devices exceeded the world population in 2014 [1]. In today's networks, mobile users have several wireless networks available to which their devices connect to (and disconnect from) automatically depending on the devices' network needs, configuration, and subscription. For example, Gao et al. [2] have shown that twenty percent of MNs have at least ten IP address changes per day, which suggests roaming between networks under different domain and/or administrative control. We define a domain as an independent network in which connecting to (one of) its wireless links by an $\mathrm{MN}$ will result in IP level mobility. LISP-MN [3] is a Locator/Identifier Split (Loc/Id [4]) protocol designed to simplify mobility between independent domains. LISP-MN ensures that data sessions are not affected by mobility event through the use of end point identifier (EID) - at the transport and upper layers of TCP/IP

This research is funded by Petroleum Technology Development Fund (PTDF), Nigeria. protocol stack - for forming communication sessions, and routing locator (RLOC) - at the network layer - for routing of packets.

While the protocol's mobility management procedure enables session continuity, it suffers three major performance limitations caused by handover delay: packet loss, service disruption; and throughput degradation as evident from the work of [5]. The delay is mostly unavoidable due to the three required handover procedures - movement detection, duplicate address detection and care-of-address configuration - as the $\mathrm{MN}$ reconnects to another access link either using its active interface or a second interface. Although the data session is not broken, packets sent as the $\mathrm{MN}$ switches to a new point of attachment (PoA) are dropped by the previous access router (AR) since it has no way of knowing where the MN has moved to.

A number of works has confirmed the negative impact of the packet loss and throughput degradation on applications. According to Biernacki [6], as small as $0.1 \%$ packet loss can cause TCP throughput to oscillate, which in turn affect the quality of video that is perceived by a user. Gorius et al. [7] reported that $0.5 \%$ loss can result in up to $25 \%$ reduction in throughput. While TCP has a mechanism to detect disconnection and slow down or stop sending packets when acknowledgements are not received, UDP-based applications will normally continue to send datagrams oblivious of the state of end-to-end links. We argue that redirecting these packets to a buffer node and forwarding the packets to the $\mathrm{MN}$ after handover will mitigate the impact of the disconnection on running applications.

To this end, our main task in this work is to present a novel network architecture that supports LISP-MN's use in multi-domain wireless environment. The new architecture is designed to mitigate the packet loss and the SDT experienced with inter-domain handovers, thereby improving the overall performance of the mobility protocol. The new architecture introduces a new network node, loc-server, and improves the LISP$\mathrm{MN}$ code to enable interaction with the server.

\section{BACKGROUND}

\section{A. LISP Mobile Node}

LISP-MN [3] is an approach defined to enable mobility with the LISP protocol. An MN is equipped 
with a lightweight version of Ingress/Egress Tunnel Router (ITR/ETR) functionality and behaves like a single LISP-domain [8]. The $\mathrm{MN}$ is configured statically with an endpoint identifier (EID - the host identity), which is used by the transport and application layer to identify communication sessions. The $\mathrm{MN}$ also configures an IP address, termed RLOC, used in determining the MN's location. A map-server (MS) is a component of LISP mapping system serving as the mobility anchor and tracks the location of the $\mathrm{MN}$ at any given time by caching EID to RLOC mapping. For communication with non-LISP correspondent node $(\mathrm{CN})$, the $\mathrm{MN}$ sends and receives all packets via a proxy ETR (PETR) and proxy ITR (PITR), respectively. The two tunnel routers are usually collocated and referred to as PXTR.

On coming online, an MN configures a new RLOC (IP address) and sends a map-register message to its MS in order to register the RLOC (its location) as shown in Fig. 1. The server will authenticate the EID in the packet and reply with a map-notify message confirming that the EID-RLOC registration has been successful and an up-to-date mapping is published on the mapping system. The MN will also send a solicit map request (SMR) message to its PITR (PXTR in the figure) - and to any LISP-based $\mathrm{CN}$ to cause the nodes to request the MN's new location. Consequently, the PITR and the $\mathrm{CN}$ would send a map-request to the $\mathrm{MN}$, to which the $\mathrm{MN}$ replies with a map-reply containing the MN's new RLOC and its EID. This ensures that the PITR and any LISP-based CN have an up-to-date location of the MN.

To establish a session with the $\mathrm{CN}$, an $\mathrm{MN}$ queries the DNS for the IP address of the host. If the MN doesn't have the mapping of the returned IP address in its cache, it sends a map-request to its map resolver and a negative reply from the mapping system indicates that the $\mathrm{CN}$ is not located in a LISP domain and it is a nonLISP device. The MN, therefore, encapsulates the outgoing packets to the PETR (PXTR) and the proxy router de-encapsulates the packets, then forwards using conventional Internet routing system. Replies are sent by the CN using the MN's EID, however, the packets would be delivered by the Internet routing infrastructure to the PITR serving the EID, which forwards the packets to the $\mathrm{MN}$.

The PITR would advertise reachability of the MN's EID prefix in the default-free zone, to enable communication between and $\mathrm{MN}$ in a non-LISP domain to another legacy host. The PITR learns of any change in the MN's location by either contacting the MS or through the SMR messages explained earlier. A nonce value is used in all LISP communications to ensure that packets received by a node are actually from the devices that claim to be sending them.

\section{RELATED WORK}

There are two major works that attempt to improve LISP-MN's control and data plane performance with focus on reducing packet loss. First is the work of Menth et al. [9] that outlines some problems with the LISP-MN including unnecessary mapping lookups for an MN in LISP domain - when an ITR performs a mapping look-up using the target MN's EID and gets the local locator (LLOC) and then looks up again using the LLOC to get the RLOC before forwarding the packet. In an attempt to mitigate this and other problems identified with the protocol, the authors proposed the introduction of local mapping system (LMS) and 'location aware' capability for MNs.

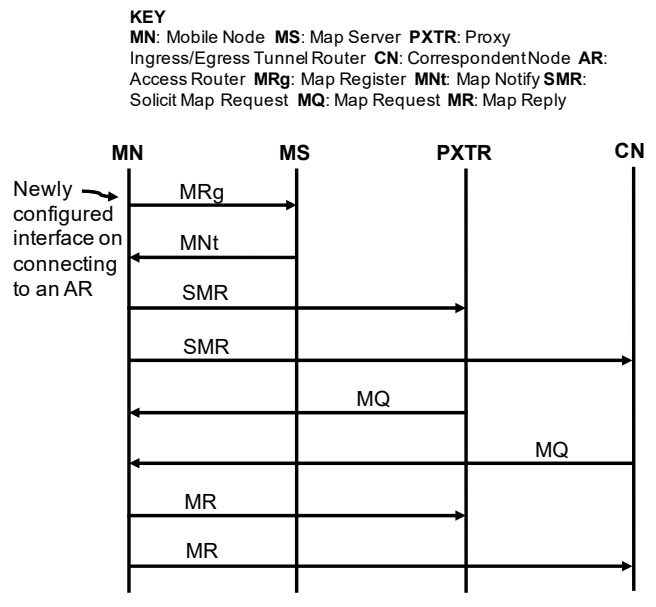

Figure 1 LISP-MN Control Plane Operation

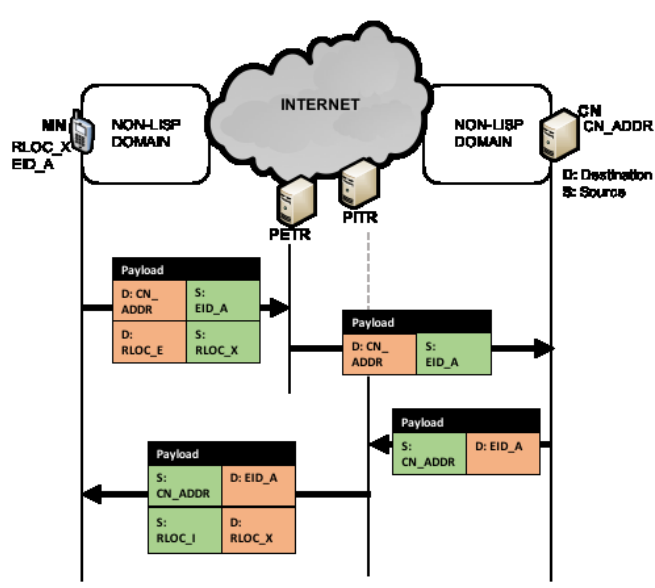

Figure 2 LISP Data Plane Operation 


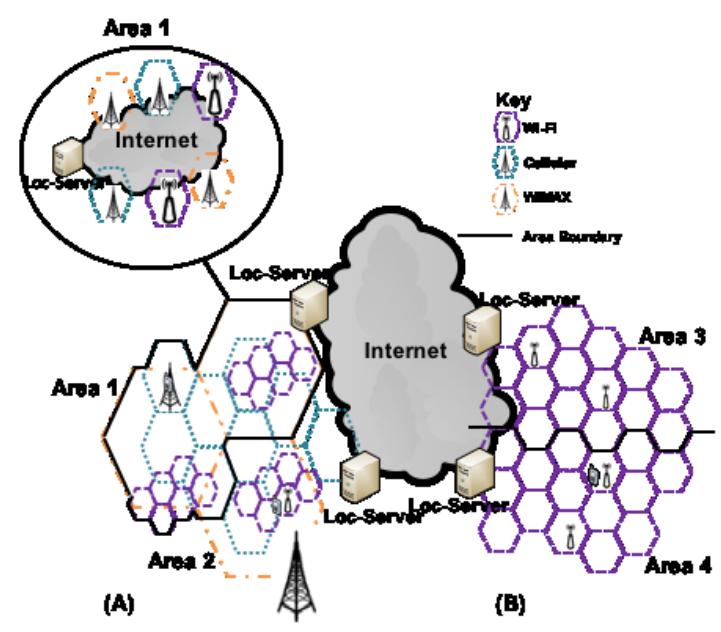

Figure 3 Location Area Server in both Heterogeneous (A) and Homogeneous (B) Wireless Environments

On connecting to a wireless link, the MN queries the mapping system with its assigned care of address $(\mathrm{CoA})$, and a negative reply indicates that it is in a nonLISP domain and the CoA is an RLOC. However, if an RLOC is returned, the CoA is a local locator (LLOC: used by MNs in LISP domain instead of RLOC) and the $\mathrm{MN}$ is in LISP domain. The MN may perform double encapsulation (LLOC packet in RLOC header) and send the packets directly to the ETR of the targeted MN and in the process avoid sending the packets to the PITR. This removes the need for the triangular routing when an $\mathrm{MN}$ in a non-LISP domain communicates with another MN in a LISP domain. However, this work is mostly focused on the data plane and the authors did not present any implementation or evaluation work.

The second work is by Gohar and Koh [10]. They proposed a network-based distributed handover control scheme, named LISP-DHC, for an MN communicating with a $\mathrm{CN}$ in the same LISP-domain. The ARs are equipped with the tunnel router functionality and maintain an EID-LLOC cache of all devices in the network. The MN's new AR performs handover signaling on behalf of the MN by exchanging maprequest and map-reply with the node's old AR, after which a bi-directional tunnel is established between the two. The new AR also exchanges the map-request and map-reply messages with the AR of the $\mathrm{CN}$, and the AR (CN) updates it EID-LLOC cache to reflect the MN's new location. CN's packets are afterwards sent directly to the MN's new location. A numerical analysis shows that the distributed scheme performs better than LISPMN and other centralized schemes such as LISP seamless mobility support scheme, and LISP proxy mobile IP, in terms of handover latency.

While the solution takes away most of the signalling from the MN (the solution is silent on MN to MS messages), the involvement of the routers means that, it could only be deployed if routers are LISPenabled, thereby limiting its deployment in real life.
There is also the need to deploy and external protocol, the Media Independent Handover (MIH) 'link-up' event to enable the routers know when an $\mathrm{MN}$ is attaching to it.

\section{IMPROVED LISP-MN ARCHITECTURE}

\section{A. Overview}

Introducing a loc-server is key to improving LISPMN's performance especially at the point of handover. The node serves as a support entity for packet delivery at the point of MN's movement. This is to eliminate or significantly reduce the amount of lost packets as the result of MN's mobility process. It is termed the locserver because it is designed to manage a defined geographical location we termed location area. A location area is a collection of homogeneous or heterogeneous wireless access technologies that are connected to a particular loc-server via the Internet (see Fig. 3). The size of a location area can be determined in a number of ways. For example, by using the potential number of devices that the server will manage. Locserver can be owned by an Internet service provider (ISP), a Telecom operator or third party service provider. The functionality could be integrated into PDN Gateway of the EPC [11] or as a separate network entity on the LISP beta network [12].

\section{B. Operation}

The proposed architecture introduces some functional modules into the LISP-based MNs which enhance the LISPmob codebase (the LISP-MN implementation [13]). This enables the $\mathrm{MN}$ to initiate interaction with the loc-server, and for the server to respond to the MN. The devices are configured with an anycast loc-server address to allow them to use the server closest to their location. This can be any area depending on the PoA that the $\mathrm{MN}$ is connected to at the time of handover. Fig, 3 depicts a topological view of location areas supported by a loc-server with the individual networks connected to the server over the Internet.

An MN intimates the MS and the PXTR of its movement by respectively sending map-register and SMR-cum-MR to the two remote nodes as discussed earlier. Since loc-server is now added to the architecture, messages are sent both prior and after a handover as shown in Fig. 4. The figure presents the interaction between the $\mathrm{MN}$ and the loc-server at both the control and the data planes. In plain LISP-MN architecture, updates are only triggered when an MN's interface is (re)configured either due to a handover or when the MN comes online. However, in the proposed architecture, mapping updates are also sent when a handover is imminent. Factors such as cost, quality of service, signal strength and/or power consumption of mobile devices can be used as handover imminent trigger (HITr). 


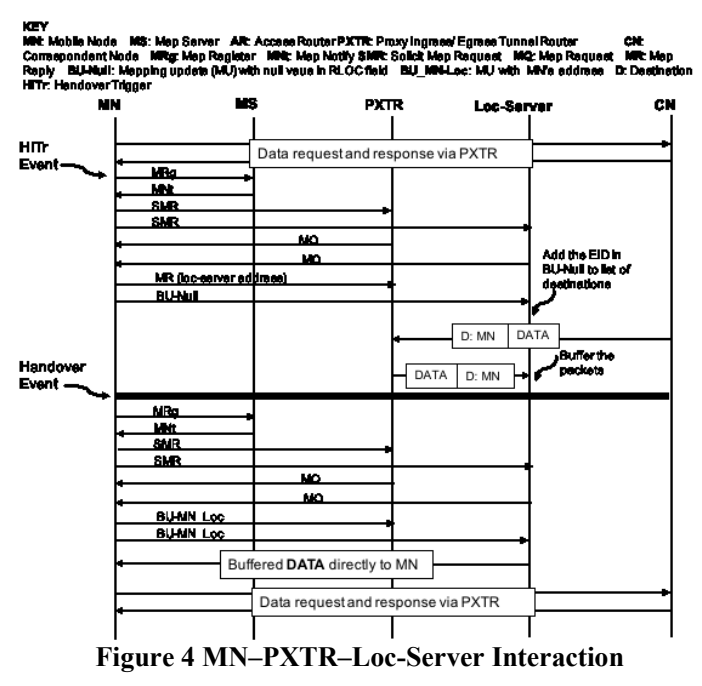

Upon a HITr, an SMR is sent to the PXTR and the loc-server for the two nodes to solicit for MN's location. The map-request generated by the two nodes prompt the $\mathrm{MN}$ to send map-reply message to both with the loc-server address in the loc field of the packet going to the PXTR. This causes the PXTR to start forwarding MN's packets to the loc-server. The mapreply to the loc-server contains a null value in the loc field and serves as a request for the server to buffer packets destined to the EID learned in the received message. The EID prefix must have been registered on the loc-server prior. At handover completion, similar map-reply is sent to the PXTR and the loc-server. This packet contains the new RLOC configured by the MN and the only difference between the packet going to the PXTR and the one going to the server is the generated nonce. The data plane resumes to status quo ante with no loc-server involvement until another HITr event.

\section{EXPERIMENTAL SETUP}

\section{A. Testbed Setup}

In order to demonstrate the improvement that our proposed architecture brings to LISP-MN's mobility event, we set up an IPv6 laboratory-based network testbed (Fig. 5). It consists of nine x86 desktop PCs running Ubuntu Linux distribution and configured accordingly as one MN, two ARs, one MS, one PXTR, one $\mathrm{CN}$, two backbone routers, and one loc-server. The systems are connected via Ethernet with the exception of the wireless links between ARs and $\mathrm{MN}$, and reachability across the network is achieved using dynamic routing with RIPv2. 802.11bgn antennas are used on all the Ubuntu desktops serving as AR/base station and $\mathrm{MN}$. There is $100 \mathrm{~ms}$ delay introduced using the Linux netem program, on the backhaul link between BR1 and BR2 to emulate the delay on the Internet. For the LISP-MN functionality on the network, we used the LISPmob implementation, running the code on the MN,

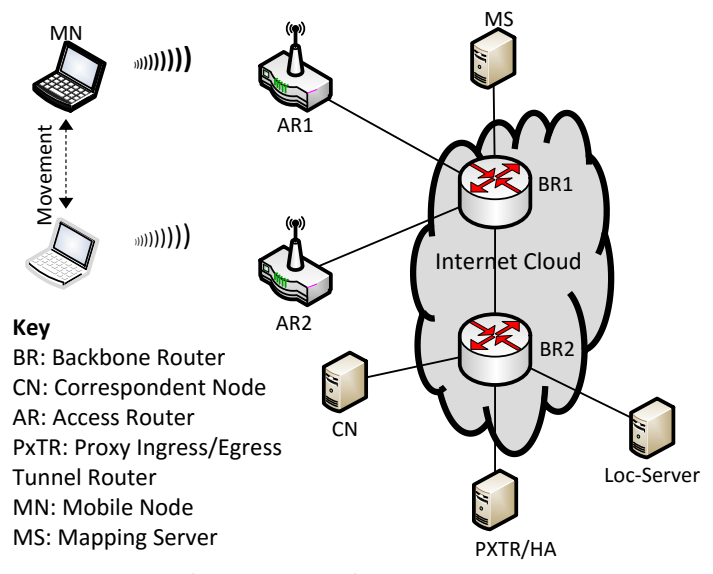

Figure 5 Experimental Testbed

the MS and the PxTR. All the systems use cubic as the TCP congestion control mechanism.

\section{B. Mobility Scenario}

Inter-domain mobility can either be vertical when an $\mathrm{MN}$ is in a heterogeneous wireless environment (HeWE) with distinct wireless technologies available; or horizontal in a homogeneous wireless environment (HoWE) where the wireless technology is of the same type. In HeWE, we consider a user connecting to a home Wi-Fi streaming a video or calling over VoIP. As soon as the user steps out and move away from the Wi$\mathrm{Fi}$, the device connects to the cellular network of his provider. We called this a hard handover as the device only switches to the cellular when the Wi-Fi is no longer available.

Similarly, on returning home, the phone discovers availability of a preconfigured Wi-Fi link and disconnects from the cellular network after connecting to the home Wi-Fi. We termed this type of switch a soft handover as the device switches to the Wi-Fi interface while the cellular interface is still up and active. Heterogeneous mobility can also be found with subscribers of Wi-Fi hotspots as they come into and leave the hotspot area. For HoWE, we are looking at a scenario where a user walks across an area with open independent Wi-Fi networks, such as a train station, malls etc, connecting and disconnecting from access points as he/she moves. The time between the start of communication and when a handover occurs is randomly set. The activity during and after the handover is the focus of the evaluation. In all our experiments, we used Wi-Fi links and interfaces to emulate cellular networks.

\section{Evaluation Metrics}

The two main improvements that the introduction of loc-server is expected to bring to LISP-MN architecture are reduction of packet loss and SDT during handover. We define the SDT as the measured difference between the time when the last packet is received before a handover and when the first packet is received after 
service reconnection. Hence the two metrics form the basis of our evaluation. For packet loss investigation, we use Linux iperf program to send UDP datagrams from a server (CN in Fig. 5) to the MN. This is in view of the fact that it is easier to capture packet loss when UDP is used because by design it does not retransmit lost packets. We emulated skype VoIP traffic over UDP with a packet size of 300 bytes to generate $64 \mathrm{kbps}$ flow to the $\mathrm{MN}$ as described in the works of [14]. For SDT, we use TCP to measure how fast the session restarts at the completion of a handover. We implemented a simple client-server program that allows the $\mathrm{MN}$ to download a file from the server over TCP, and we use the traffic traces from this communication to measure the SDT.

We run experiments for both HeWE and HoWE using fast and slow wireless links to thoroughly test the new architecture in different wireless conditions. We consider fast links to be radio links that have more than $2 m b p s$ download capacity, while slow link is considered to be a channel with less than $2 m b p s$. This is based on the work of Riiser et. al [15] which shows that $2 \mathrm{mbps}$ bandwidth is rarely attained when streaming media over $3 \mathrm{G}$ while commuting on metro, bus, tram, train or ferry in Oslo suburb. 4G networks provides more than $2 \mathrm{mbps}$ download on average as evident from OFCOM UK 2015 statistical data [16]. Hence evaluating two different link speeds is vital.

To test the impact of the new architecture on applications, the throughput-based DASH player [17, 18 ] is used over a HoWE to see the performance of video applications on the networks. We set up a webserver using Apache 2.4.17, which hosts the video dataset and the open source film Big Buck Bunny from [19] is used. It consists of different quality levels, from 50 to 5,000kbps, that are available for an $\mathrm{MN}$ to choose from the server. The results presented in section VI are averages of 10 experimental run unless otherwise specified.

\section{EXPERIMENTAL RESULTS}

\section{A. Packet Loss}

The percentage of lost packets in both heterogeneous and homogeneous environments and for the different link speeds is given in Tables 1 and 2. For HeWE, 2,055 datagrams were sent to the $\mathrm{MN}$ by the server in the period of 65 seconds with hard and soft handovers conducted within this period. There is $49 \%$ more lost packets on Plain LISP in comparison to Loc_LISP. In other words, the new architecture halved the number of dropped packets in the network for both fast and slow links. As observed from Fig. 6 (A \& B), all the packet losses were recorded during the $\mathrm{HH}$ event (started at $23^{\text {rd }}$ second) as no sign of disruption was recorded for the soft handover event at $47^{\text {th }}$ second. For
Table 1 Packet Loss in Heterogeneous Environment

\begin{tabular}{|c|c|c|c|}
\hline Links & Loc_LISP \% & Plain_LISP \% & Difference \% \\
\hline Fast & 7.64 & 15 & 49 \\
\hline Slow & 7.64 & 15 & 49 \\
\hline
\end{tabular}

Table 2 Packet Loss in Homogeneous Environment

\begin{tabular}{|c|c|c|c|}
\hline Links & Loc_LISP \% & Plain_LISP \% & Difference \% \\
\hline Fast & 3.98 & 17 & 77 \\
\hline Slow & 4 & 17.85 & 81 \\
\hline
\end{tabular}

the HoWE however, 1,788 datagrams were sent by the server over the period of one minute with one handover started at $29^{\text {th }}$ second. Plain_LISP, is this case, suffered four times more losses than Loc LISP where $77 \%$ of the packets sent during handover where saved and delivered to the MN whilst on the fast link and $81 \%$ on slow for the Loc LISP. The better performance shown by MN on Loc_LISP in both HeWE and HoWE is expected as the loc-server saves and forwards all the packets to the $\mathrm{MN}$ on handover completion.

On observation of packet traces on the loc-server, we realised that it saves and forwards all packets sent to it by the PXTR as the MN changes PoA. Hence, the lost packets in the case of Loc_LISP scenarios are likely caused by two events: the burst of traffic from the locserver combined with traffic coming from the PXTR immediately after the handover are too much for the MN's network interface card to handle and packets were dropped at that point; and/or there are packets on the wire from the PXTR that arrived at the previous AR after the $\mathrm{MN}$ had already began the handover process and were dropped by the AR since the $\mathrm{MN}$ was no more connected to it.

\section{B. Service Disruption Time}

For SDT, Loc_LISP consistently shows better performance in all the four scenarios as presented in Table 3 . The average handover delay recorded for hard handover event in HeWE is measured at about 3.27s, which shows that the session resumes immediately the movement event is completed $(3.3 s$ for fast link and $3.7 s$ for slow). This is in contrast to the Plain LISP that recorded $5.6 s$ for fast link and $6 s$ for slow. Loc_LISP achieved about $40 \%$ reduction in SDT in HeWE. It is a similar story for HoWE where Loc LISP also showed shorter SDTs in comparison to the Plain LISP. The effect can be clearly seen on the throughput graphs of Fig. 6. The handover caused disruption of service as the throughput went to zero during the process. With Loc_LISP though, it comes back up as soon as the handover is completed as shown in the figure, contrary to the Plain_LISP which takes some time to re-establish the session in all the four scenarios. 

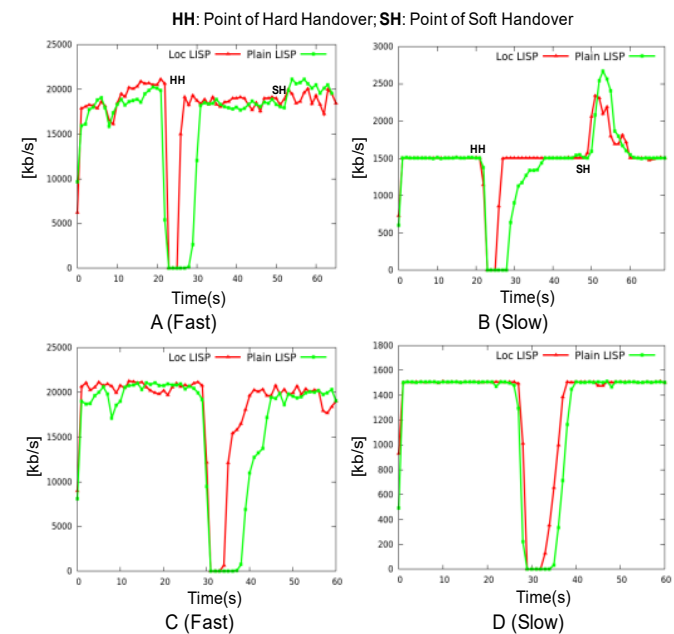

Figure 6 TCP Throughput for HeWE (A \& B) and HoWE (C \& D) Showing Effect of Handover

Table 3 Average service disruption time

\begin{tabular}{|c|c|c|c|c|}
\hline \multirow[b]{2}{*}{ Protocols } & \multicolumn{2}{|c|}{$\begin{array}{l}\text { Heterogeneous( } \\
\text { secs) }\end{array}$} & \multicolumn{2}{|c|}{$\begin{array}{l}\text { Homogeneous } \\
\text { secs) }\end{array}$} \\
\hline & Fast & Slow & Fast & Slow \\
\hline $\begin{array}{l}\text { Loc } \\
\text { LISP }\end{array}$ & 3.3 & 3.7 & 3.7 & 4.1 \\
\hline $\begin{array}{l}\text { Plain_- } \\
\text { LISP }\end{array}$ & 5.6 & 6.0 & 6.1 & 6.0 \\
\hline
\end{tabular}

Soft handover for slow link in HeWE saw a temporal rise in throughput (Fig. 6B) as data is delivered via the two interfaces momentarily before the switch is completed. There was no similar rise in the fast link likely because, either the TCP congestion window at the $\mathrm{CN}$ or the MN's receive window is already at its maximum, leaving no room for improvement. During the course of the handover, the $\mathrm{CN}$ stops sending TCP packets to the MN after a period of no packet acknowledgement from the MN. At the completion of handover, the transport layer realizes the availability of a new connection and then starts sending acknowledgement. With packets coming from the loc-server in Loc_LISP immediately after the handover, the MN's transport layer instantly requests for the next packets from the $\mathrm{CN}$ without any delay prompting the quick resumption of the data session.

\section{Video Applicaton Performance}

To measure the performance of the DASH player in a HoWE, the MN streams video from the server for about $150 \mathrm{~s}$ conducting four handovers in the process. This is to enable us to measure the impact that the movements have on the average quality that the user downloaded and the instability caused by the handover.

As shown in Fig. 7, the player running on the Loc_LISP recorded higher quality level for fast link as handovers were executed on the $30^{\text {th }}, 53^{\text {rd }}, 76^{\text {th }}$ and $99^{\text {th }}$
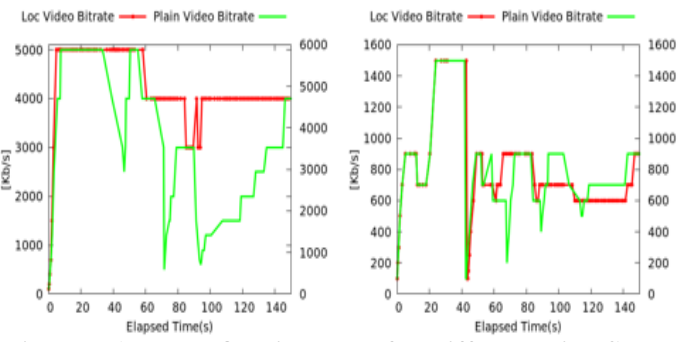

Figure 7 Average Quality Level for Different Link Speeds

seconds. The player was not affected until the second handover was started, in contrast to the Plain_LISP where the quality level drops immediately after the first handover was executed and stayed low till the end of the streaming session. Even when the handovers were executed every $23 s$, Loc LISP recorded high quality level owing to the fact that packet loss is significantly reduced.

Furthermore, the average video rate of the player is improved on the fast link when the proposed architecture is used. An average of 4,055kbps was recorded for Loc_LISP against 3,042kbps on Plain_LISP architecture. On the contrary, Loc_LISP did not show any improved video rate performance on slow links with similar average video rate recorded for the two architectures - 820kbps against 821kbps for Plain LISP.

While there is no significant difference in the average video rate and video quality level on slow link between Loc_LISP and Plain_LISP, the former architecture enables the player to have a more stable download as the player adjusts its video rate request due to the changes at the network layer caused by the handovers. As observed in Fig. 8, percentage instability (the rate at which a different video quality is downloaded) of Plain_LISP is higher than Loc_LISP especially for the fast link. These frequent changes in quality are known to affect the quality of experience of a user in a negative way. Hence a user on the Loc_LISP architecture streaming a video on slow link will still have better viewing experience than playing

LOC_LISP $\square$

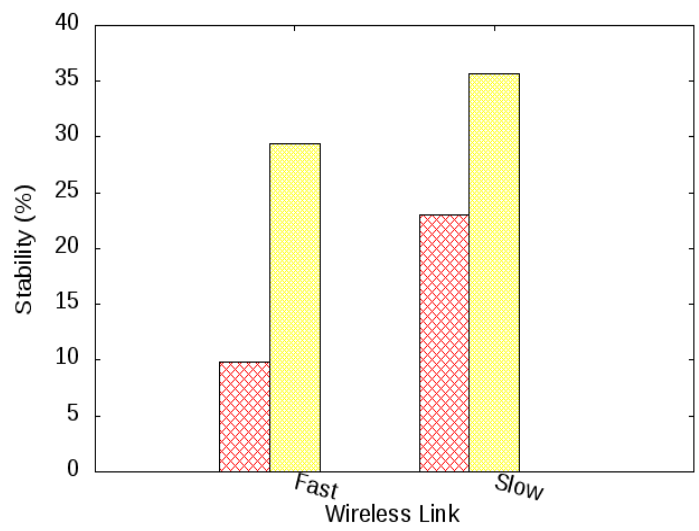

Figure 8 Stability: Percentage Change in Video Quality 
the video on Plain_LISP.

\section{CONCLUSION}

The LISP-MN's long service disruption time (SDT) and packet loss cause degradation in network performance for mobile devices. In this work, we proposed an improved architecture for LISP-MN (Loc_LISP) that significantly reduces the SDT and the packet loss experience by mobile nodes (MN) at the point of handover. The proposed scheme introduces a new node into LISP-MN's architecture, termed locserver, which is responsible for buffering packets that are dropped at the point of handover. Experiments were run on a laboratory testbed for different link speeds. We found that by introducing the loc-server packet losses are significantly reduced. We also recoded significant reduction in the SDT as the MN is spurred by the arriving buffered packets to immediately request for subsequent packets from the server rather than wait a little longer as we observed with Plain_LISP. The effect of this improvement is

\section{REFERENCES}

[1] "Cisco Visual Networking Index: Global Mobile Data Traffic Forecast Update 2014-2019 White Paper," Cisco Systems Inc., San Jose, Feb., 2015.

[2] Z. Gao, A. Venkataramani, J. Kurose, and S. Heimlicher, "Towards a Quantitative Comparison of the Cost-Benefit Trade-offs of Location-Independent Network Architectures," in ACM SIGCOMM'14, Chicago, IL, USA, 2014.

[3] C. White, D. Lewis, D. Meyer, and D. Farinacci, "LISP Mobile Node," in IETF Internet Draft, draft- meyerlisp-mn-16, Jul. 2016.

[4] W. Ramirez, X. Masip-Bruin, M. Yannuzzi, R. SerralGracia, A. Martinez, and M. S. Siddiqui, "A survey and taxonomy of ID/Locator Split Architectures," Computer Networks, v.60, no. 0, pp. 13-33, 2/26/ 2014.

[5] M. Isah and C. Edwards, "Inter-domain Mobility with LISP-MN--A Performance Comparison with MIPv6," in 2015 8th IFIP Wireless and Mobile Networking Conference (WMNC), Munich, 2015. pp. 80-87: IEEE.

[6] A. Biernacki and K. Tutschku, "Performance of HTTP video streaming under different network conditions," Multimedia Tools and Applications, vol. 72, no. 2, pp. 1143-1166, 2013.

[7] M. Gorius, S. Yongtao, and T. Herfet, "Dynamic media streaming over wireless and mobile IP networks," in Consumer Electronics - Berlin (ICCE-Berlin), 2012 IEEE International Conference on, 2012,. pp. 158-162.

[8] V. Fuller and D. Farinacci, "Locator/ID Separation Protocol (LISP) Map-Server Interface," in IETF RFC 6830, Jan. 2013.

[9] M. Menth, D. Klein, and M. Hartmann, "Improvements to LISP Mobile Node," in Teletraffic Congress (ITC), 2010 22nd International, 2010, pp. 1-8.

[10] M. Gohar and K. Seok-Joo, "Distributed handover control in localized mobile LISP networks," in Wireless and Mobile Networking Conference (WMNC), 2011 4th Joint IFIP, 2011, pp. 1-7. also visible on a DASH video player, which recorded higher average video quality and improved stability as the amount of lost packets in the Loc_LISP environment is significantly mitigated. We have also realised that while our solution improves LISP-MN performance for slow links, it has greater impact on links that are greater than 2 mbps. The additional control messages of the new architecture, in comparison to the LISP-MN architecture, may potentially incur extra computational cost in large scale deployment, however, we have not noticed any variation in performance between the two architectures running on the laboratory testbed used for the experiments.

\section{VIII.FUTURE WORK}

Our next line of work is to integrate the loc-server functionality into the PXTR node to reduce the pathstretch of the packets sent during a handover. This will also reduce, by $50 \%$, the number of control messages introduced in the new architecture.

[11] Universal Mobile Telecommunications System (UMTS); LTE; Access to the 3GPP Evolved Packet Core (EPC) via non-3GPP access networks; Stage 3 (3GPP TS 24.302 version 11.7.0 Release 11), 2013.

[12] (2016, 12/06/2016). LISP Beta-Network. Available: http://www.lisp4.net/beta-network/.

[13] (2014, 09/01/2014). LISPmob - an open-source LISP implemantation for Linux, Android and OpenWRT. Available: http://lispmob.org/

[14] D. Phoomikiattisak, "Mobility as first class functionality: ILNPv6 in the Linux kernel," PhD Diss., University of St Andrews, 2016.

[15] H. Riiser, P. Vigmostad, C. Griwodz, and P. Halvorsen, "Commute path bandwidth traces from $3 \mathrm{G}$ networks: analysis and applications," in Proceedings of the 4th ACM Multimedia Systems Conference, 2013, pp. 114-118: ACM.

[16] Ofcom, "Measuring Mobile Broadband Performance in the UK," 2015, Available: http://stakeholders.ofcom.org.uk/binaries/research/broa dband-

research/april15/Ofcom_MBB_Performance_Report_ April_2015.pdf

[17] (2014, 08/03/2014). Microsoft Smooth Streamer Open Source. Available: https://slextensions.svn.codeplex.com/svn/trunk/SLExt ensions/AdaptiveStreaming/

[18] S. Akhshabi, A. C. Begen, and C. Dovrolis, "An experimental evaluation of rate-adaptation algorithms in adaptive streaming over HTTP," in Proceedings of the second annual ACM conference on Multimedia systems, 2011, pp. 157-168: ACM.

[19] S. Lederer, C. Müller, and C. Timmerer, "Dynamic adaptive streaming over HTTP dataset," in Proceedings of the 3rd Multimedia Systems Conference, 2012, pp. 89-94: ACM. 